\title{
Los recursos digitales del Museo Nacional de Ciencias Naturales durante el confinamiento por COVID-19 y su evaluación
}

\section{PREMIIO CIMED}

\author{
The digital resources of the Museo Nacional de Ciencias Naturales \\ during lockdown and their evaluation
}

Versión en español, *English version at Anexo I

\begin{abstract}
Pilar López García-Gallo , Eloísa Pérez Santos ${ }^{\mathrm{b}}$, Rocío de Iriarte Rodríguez ${ }^{\mathrm{c}}$, Azucena López Márquez ${ }^{\mathrm{d}}$, Marta Fernández Lara ${ }^{\mathrm{e}}$.

${ }^{a}$ Museo Nacional de Ciencias Naturales (MNCN-CSIC) pilarg@mncn.csic.es, Universidad Complutense de Madrid y Laboratorio Permanente de Público de Museos, eperezsa@ucm.es, ${ }^{c}$ Museo Nacional de Ciencias Naturales (MNCN-CSIC) rociodeiriarte@mncn.csic.es, ${ }^{\mathrm{d}}$ Museo Nacional de Ciencias Naturales (MNCN-CSIC) azucenalopez@mncn.csic.es, ${ }^{\mathrm{e}}$ Museo Nacional de Ciencias Naturales (MNCN-CSIC) martaflara@mncn.csic.es.
\end{abstract}

\begin{abstract}
Resumen
El Museo Nacional de Ciencias Naturales (MNCN-CSIC), consciente del papel creciente del entorno digital en los museos, especialmente tras la crisis por COVID-19, incluyó una sección de recursos digitales en su página web antes de la declaración del estado de alarma, periodo en el que estos recursos se potenciaron y ampliaron mediante la creación de actividades didácticas online, juegos digitales y juegos online, vídeos divulgativos, difusión de exposiciones y visitas virtuales realizadas anteriormente junto a Google Arts \& Culture. En cuanto a las redes sociales, el objetivo fue acercar las colecciones, la investigación y las exposiciones a los usuarios. Durante la segunda quincena de marzo de 2020, y al inicio del estado de alarma y confinamiento, se notó un incremento en el interés de las publicaciones en RRSS respecto a las realizadas antes de la pandemia. Entre marzo y junio de 2020 se comprobó que la comunidad de seguidores en redes sociales del MNCN interactuó notablemente y comentó más los contenidos propuestos. Además, del 14 de marzo al 28 de mayo la página web del MNCN recibió 284.848 visitas. Un total de 98.303 usuarios consultaron el contenido online del Museo. El confinamiento también supuso una oportunidad para evaluar la recepción y la efectividad de los recursos digitales iniciados y recabar información relevante que permitiera adelantarse a las inquietudes e intereses del público, a través de un cuestionario online realizado en mayo de 2020, durante la fase 1 de la desescalada y justo antes de la reapertura de equipamientos culturales y museos. También se evaluó la predisposición a volver
\end{abstract}


a las salas del Museo, la disponibilidad para seguir actividades en streaming en el futuro previo pago y otros sistemas de financiación de contenidos online.

Los resultados mostraron que estos contenidos fueron muy valorados por los encuestados que realizaron un seguimiento de estos de forma proactiva, ya que casi la mitad afirmó seguir la información digital publicada por el MNCN. Los visitantes más habituales, aquellos que habian realizado varias visitas presenciales en los últimos 2 años, fueron los que más información recordaron haber visto y buscado.

Palabras Clave: confinamiento; pandemia, recursos digitales; redes sociales; evaluación; cuestionario online, museo

\begin{abstract}
The Museo Nacional de Ciencias Naturales (MNCN-CSIC), aware of the growing role of digital environments in museums intensified by the COVID-19 crisis, included a section of digital resources on its website before the state of emergency was declared. During this time, these resources were increased and improved through the creation of online educational activities: online and digital games, informative videos, the dissemination of exhibitions, and virtual visits previously created with Google Arts \& Culture. In terms of social networks, the objective was to bring collections, research, and exhibitions closer to users. During the second half of March 2020 and at the beginning of the state of emergency and lockdown, interest in the publications on social networks increased. Between March and June 2020, the community of MNCN followers on social networks interacted with and commented significantly more on the content. In addition, from March 14 to May 28, the MNCN website received 284,848 visits. A total of 98,303 users consulted the Museum's online content. Lockdown also provided us with an opportunity to evaluate the reception and effectiveness of our digital resources and collect relevant information that would allow us to anticipate the concerns and interests of the public. We posted an online questionnaire in May 2020, during phase 1 of reopening and just before cultural facilities and museums were to open to the public. People's willingness to return to the Museum, to participate in paid streaming activities in the future, and other online content financing systems were also evaluated. The results showed that these contents were highly valued by the respondents who proactively used them, as almost half claimed to follow the digital information published by the MNCN. The most frequent visitors, those who had made several face-to-face visits in the last two years, were the ones who remembered seeing and searching for the most information.
\end{abstract}


Pilar López García-Gallo, Eloísa Pérez Santos, Rocío de Iriarte Rodríguez, Azucena López Márquez, Marta Fernández Lara

Keywords: lockdown; pandemic, digital resources; social media; evaluation; online questionnaire, natural history museum

Este trabajo de investigación se ha realizado a iniciativa de la Vicedirección de Comunicación y Cultura Científica del MNCN con la colaboración de la Vicedirección de Exposiciones y con la asesoría cientifica para el diseño de la investigación de Eloísa Pérez Santos (Universidad Complutense de Madrid). El formato y los contenidos del cuestionario se diseñaron de forma colaborativa y bajo el auspicio de la Dirección del MNCN-CSIC y del Laboratorio Permanente de Público de Museos (SGME). 


\section{Introducción}

El cierre de los museos y el confinamiento de la población desencadenada por la emergencia sanitaria de la COVID-19 ha puesto de manifiesto las debilidades de las estrategias digitales de estas instituciones además de plantear numerosos interrogantes de cara a una futura, aunque cuestionable, normalidad.

El Museo Nacional de Ciencias Naturales (MNCN-CSIC), como todos los espacios culturales del país, permaneció cerrado por la pandemia desde mediados de marzo hasta principios de junio de 2020. Esta circunstancia activó los planes de digitalización que ya habían comenzado en últimos años en el Museo y se convirtió en una oportunidad para lanzar nuevas propuestas digitales tanto para los visitantes presenciales que, durante esos meses no pudieron acudir al Museo, como para una audiencia virtual más amplia que estuviera interesada en este tipo de contenidos.

La Vicedirección de Comunicación y Cultura Científica del Museo, consciente del papel creciente del entorno digital en los museos, visibilizó la oferta online que llevaba realizando durante años y presentó nuevas propuestas digitales para que el público pudiese seguir disfrutando de la ciencia a través del Museo durante la cuarentena y, tras ella desde cualquier parte del país y fuera de él, desde sus hogares. En la página web, que desde su lanzamiento en octubre de 2019 hasta febrero de 2020 había recibido una media de 170.000 visitas, se incluyó una sección de recursos digitales antes de la declaración del estado de alarma, periodo en el que estos recursos se potenciaron y ampliaron. Se realizó la primera exposición virtual elaborada en colaboración con el público seguidor del Museo: "Naturaleza artificial: Vuestra exposición", en la que el hilo argumental ha sido la biodiversidad que encontramos en nuestros hogares, pero cuyo verdadero núcleo son las propias historias contadas por el público que se convirtió en co-creador de la muestra. La convocatoria se hizo a través de redes sociales para que los seguidores enviaran imágenes de animales artificiales. La exposición se inauguró el 4 de mayo de 2020, estando el museo cerrado al público: [https://www.mncn.csic.es/es/visita-el-mncn/exposiciones/naturaleza-artificial-vuestraexposicion]. El 27 de octubre de 2020 se presentó, de forma presencial y en streaming, al profesorado de educación primaria el proyecto 'Cuéntame cómo dedicarme a la ciencia', un recurso educativo que, a través de audiocuentos, pretende acercar la ciencia y la figura de mujeres investigadoras del museo a los más pequeños. Además de 10 narraciones y la canción 'Quiero investigar', el proyecto incluye una guía educativa con ilustraciones originales, propuestas para trabajar en el aula, biografías de las protagonistas y explicaciones de algunos de los conceptos que se tratan en cada historia [https://www.mncn.csic.es/es/visita-elmncn/educacion/cuentame-como-dedicarme-la-ciencia].

Así mismo, la imposibilidad de abrir las puertas del MNCN durante el estado de alarma, hizo que se replanteara la estrategia de Comunicación y Cultura Científica de esta institución a través de sus redes sociales. Los anuncios de las actividades para todos los públicos, de las conferencias o de las exposiciones temporales tuvieron que ser sustituidos por contenidos digitales; visitas virtuales, exposiciones online de Google Arts, información de archivo sobre 
Pilar López García-Gallo, Eloísa Pérez Santos, Rocio de Iriarte Rodríguez, Azucena López Márquez, Marta Fernández Lara

colecciones, recursos educativos online (existentes y nuevos), juegos digitales. [https://www.mncn.csic.es/es/visita-el-mncn/actividades/recursos-digitales ].

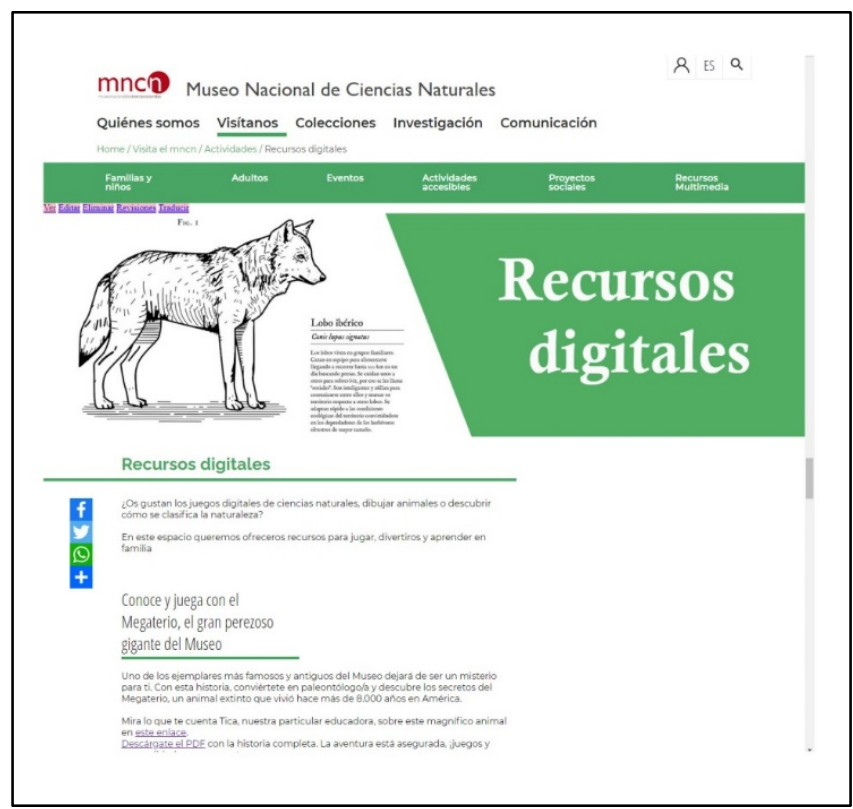

Fig. 1 EI MNCN cuenta con distintos espacios en su web donde se agrupan los recursos digitales

Al mismo tiempo, el Museo tuvo en cuenta que, como algunos autores importantes han venido señalando (Villaespesa, 2018), la experiencia digital en los museos ha ido evolucionando en los últimos años, pasando de una interacción estática con los usuarios a una caracterizada por la comunicación bidireccional y la participación en múltiples plataformas. Por ello, se decidió crear publicaciones que mostrasen y acercasen las colecciones, la investigación y las exposiciones a los usuarios a través de los contenidos digitales disponibles, realizándose, entre marzo y mayo de 2020 unas 300 publicaciones en los perfiles de las redes sociales del Museo (Facebook, Twitter e Instagram), cuidándose especialmente la interacción con el público que reaccionaba a esos contenidos y siguiendo en todo momento las estrategias de diseminación que hacen un uso óptimo de los canales y recursos de comunicación científica disponibles (Dudo y Besley, 2016; Valero y García (2018). 
Los recursos digitales del Museo Nacional de Ciencias Naturales durante el confinamiento por COVID-19 y su evaluación

Tabla 1. Recursos digitales y número de publicaciones del MNCN de marzo a junio de 2020

\begin{tabular}{|c|c|c|}
\hline Recursos & Dirección & № publicaciones \\
\hline Página web & $\underline{\text { www.mncn.csic.es }}$ & 7 \\
\hline Twitter & https://twitter.com/MNCNcomunica & 143 \\
\hline Facebook & https://www.facebook.com/MNCNcomunica & 118 \\
\hline Instagram & https://www.instagram.com/museocienciasmadrid/?hl=es & 108 \\
\hline Newsletters & $\begin{array}{l}\text { https://us11.admin.mailchimp.com/campaigns/wizard/neapolitan?id= } \\
\underline{727961} \\
\underline{\text { https://us11.campaign- }} \\
\underline{\text { archive.com/?e=[UNIQID]\&u=793285be5810fa0c4613bf9d0\&id=5e44] }} \\
\underline{6 f f 6} \\
\underline{\text { https://us11.campaign- }} \\
\underline{\text { archive.com/?e=[UNIQID]\&u=793285be5810fa0c4613bf9d0\&id=0e72. }} \\
\underline{4719} \\
\text {...entre otras... }\end{array}$ & 10 \\
\hline Youtube & https://www.youtube.com/channel/UCXMQC8IOSuQuHiPXgk60wHA & 4 \\
\hline
\end{tabular}

El interés por conocer cómo toda esta actividad digital fue recibida por el público del Museo y la necesidad de conocer, antes de la reapertura, la disposición a volver presencialmente a las salas expositivas y/o, en su caso, mantener un seguimiento online de los recursos ya iniciados, fue el detonante para iniciar un estudio sobre el tema. Esta investigación inicial se enmarca dentro de la Unidad de Estudios de Público del Museo Nacional de Ciencias Naturales, creada en 2019, para investigar los perfiles de los visitantes y audiencias digitales; sus intereses, necesidades y sugerencias de mejora, así como la efectividad de los recursos y servicios del museo. [https://www.mncn.csic.es/es/visita-el-mncn/actividades/unidad-deestudios-de-publico-del-mncn].

\section{Objetivos}

El objetivo principal del trabajo que se presenta fue la evaluación del nivel de seguimiento e interés en los recursos digitales lanzados por el Museo durante el confinamiento, después de su cierre y durante el estado de alarma decretado por la pandemia mundial por COVID-19 y 
relacionarlo con la disposición, temores y necesidades de los visitantes del Museo Nacional de Ciencias Naturales (MNCN-CSIC) ante la reapertura del Museo1.

Este objetivo general se concretó en una serie de objetivos específicos que permitieran dar voz a los visitantes y escuchar sus preocupaciones ante la reapertura del Museo, así como recabar información relevante que permitiera adelantarse a sus inquietudes e intereses:

Realizar un análisis de resultados de los recursos digitales del Museo a lo largo del tiempo, antes, durante y después del cierre, confinamiento y reapertura.

Conocer el seguimiento por parte del público de los recursos y contenidos digitales que el Museo puso en marcha o potenció durante el periodo de confinamiento.

Investigar la opinión del público sobre estos contenidos, su predisposición a volver de forma presencial en esta nueva etapa y a seguir manteniendo su vinculación con los nuevos recursos digitales del Museo.

Este trabajo se ha elaborado con los datos recogidos en un primer estudio inicial de una serie de investigaciones que el MNCN realizará, durante los próximos años, sobre las características, motivaciones y opiniones de sus visitantes, así como de su experiencia presencial y digital de la visita y que servirán para conectar aún más el Museo con su público.

\section{Metodología}

Como afirma Villaespesa (2018) para poder tener una visión completa del usuario, se necesita combinar distintos tipos de herramientas y métodos de recogida de información, por lo que los datos proporcionados por analíticas web deben ser cumplimentados con otros sobre motivaciones, expectativas, necesidades y satisfacción asequibles a través de otro tipo de estrategias como los cuestionarios y las entrevistas al público. Por ello, la metodología utilizada en este trabajo para evaluar los objetivos propuestos fue múltiple intentando relacionar los datos cuantitativos procedentes de las métricas digitales con los obtenidos a través de cuestionarios y entrevistas al público.

Por un lado, se utilizaron analíticas digitales para la medición del uso e impacto de los recursos digitales del Museo antes y después del confinamiento, fundamentalmente, Google Analytics y análisis de redes sociales. Para observar y medir los nuevos seguidores, las visitas al perfil, las impresiones, las interacciones, los comentarios, las visualizaciones, el alcance y las menciones, se usaron las propias herramientas proporcionadas e incluidas en las redes sociales analizadas de los perfiles activos del MNCN: Twitter, Facebook e Instagram. Para ello, se utilizó la herramienta del calendario para conocer los datos en los periodos concretos de interés. Además, se realizó un informe mensual (desde enero de 2020 hasta la actualidad) con los siguientes indicadores: nuevos seguidores en cada red social, número de

\footnotetext{
${ }^{1}$ Los resultados completos de la evaluación realizada a través del cuestionario online, pueden consultarse en https://www.mncn.csic.es/sites/default/files/2020-09/DosierEstudioPublico_FINAL.pdf
} 
publicaciones total y porcentaje de publicaciones sobre las diferentes temáticas tratadas en el MNCN.

Google Analytics fue la herramienta empleada para la toma de datos relacionada con la página web (www.mncn.csic.es). Gracias a ella se pudieron analizar mensualmente (desde su lanzamiento en octubre de 2019 hasta la actualidad) 14 parámetros diferentes: visitas a páginas, sesiones, usuarios, porcentaje de rebote, duración media de la sesión, media de páginas visitadas por sesión, visitas por países, visitas por comunidades autónomas, dispositivos usados, sexo y edad de los usuarios, usuarios nuevos vs. recurrentes, adquisición y páginas más vistas. Esto ha permitido ver la evolución de la página web y los cambios y diferencias que la pandemia ha provocado en el comportamiento habitual de las personas que nos visitaban, tanto las nuevas como las recurrentes, en cuanto a sus búsquedas, procedencia de las visitas y contenidos más visitados.

También se diseñó un cuestionario online que se dio a conocer a través de listas de distribución por correo electrónico, la propia página web del museo y sus perfiles en redes sociales (Facebook, Twitter e Instagram), enviándose a reconocidos divulgadores y blogueros científicos y culturales (Twitter, Instagram, etc.) para que pudieran difundirlo. Este cuestionario fue diseñado a partir de la experiencia de trabajos anteriores del equipo de planificación de esta investigación (Pérez Santos, 2009; 2018; 2020) con la participación del personal de la Vicedirección de Exposiciones y de la Dirección del centro, siguiendo un proceso colaborativo y participativo, involucrando a todos los participantes en su desarrollo e implementación, lo que, como demuestra la experiencia, elimina el temor asociado a todo proceso evaluativo que en muchas instituciones todavía persiste (Pérez Santos, 2019).

Por último, se llevaron a cabo algunas entrevistas cortas a los visitantes presenciales el día de la reapertura del museo y se analizó la asistencia a actividades durante el verano y otoño de 2020, después de que el Museo volviera abrir de nuevo.

El cuestionario online tenía como objetivo investigar a un público amplio que conocía presencial o virtualmente, en mayor o menor medida, el Museo Nacional de Ciencias Naturales, sobre el seguimiento y la satisfacción con sus recursos digitales, además de otras variables de interés de cara a la reapertura. Para ello se lanzó entre el 13 y el 28 de mayo de 2020, durante la fase 1 de la desescalada y justo antes de la reapertura de equipamientos culturales y museos.

A través del procedimiento de encuesta, el cuestionario fue respondido por 1.122 personas, las cuales resultaron ser visitantes más o menos habituales del Museo (el 83\%), muchas (el $23,2 \%$ ) habiendo realizado varias visitas en los últimos años, principalmente con niños (el $25,6 \%$ ), para participar en talleres, conferencias, etc. El 63,7\% eran mujeres y el 81,3\% tenían edades comprendidas entre los 30 y los 65 años. Un $85 \%$ poseían estudios superiores y aproximadamente la mitad, el 49,7\%, una formación y/o profesión relacionada con el ámbito científico. Casi todos, el 82,6\%, eran residentes en Madrid. 


\section{Resultados}

Uso e impacto de los recursos digitales del Museo y redes sociales durante el cierre del Museo en el confinamiento

En la segunda quincena de marzo de 2020, y coincidiendo con el inicio del estado de alarma y confinamiento, se notó un incremento en el interés de las publicaciones digitales. Concretamente del 14 de marzo, fecha de inicio del estado de alarma, al 28 de mayo, día de cierre de esta encuesta online administrada, la página web del Museo Nacional de Ciencias Naturales de Madrid recibió 284.848 visitas a sus páginas. Un total de 98.303 usuarios consultaron el contenido online del Museo. De este total de usuarios, el 11,93\% fueron recurrentes frente a un $88,07 \%$ que fueron nuevos usuarios.

Aunque al principio del confinamiento (del 15 de marzo al 15 abril), se aprecia una caída acusada de visitas con solo 95.915, una de las cifras más bajas registradas en todo el año, a partir de abril hay un crecimiento del $32 \%$, que luego se va estabilizando, coincidiendo con la publicación de los recursos digitales creados durante el confinamiento, con el Museo ya cerrado.

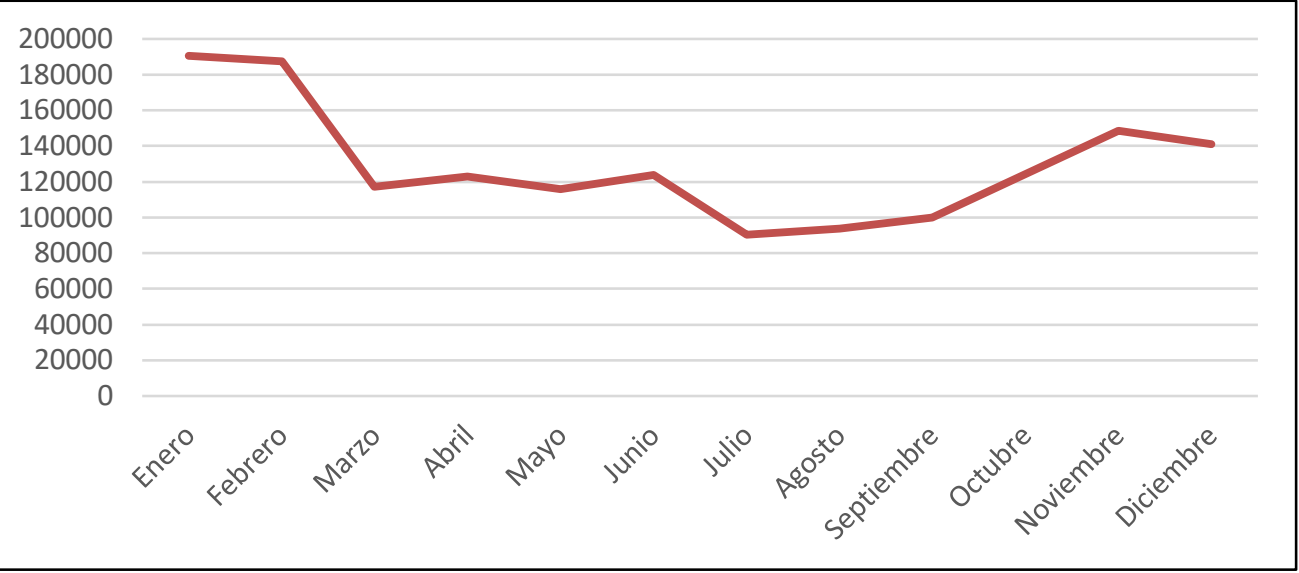

Fig. 2 Evolución del número de visitas totales a páginas web del MNCN durante 2020

Un dato curioso es que mientras que desde el lanzamiento de la página web en 2019 hasta febrero de 2020 el 52,48\% de las visitas a la página procedían de Madrid, durante el confinamiento, este porcentaje decrece hasta el $26,4 \%$ en marzo y el $27 \%$ en abril en favor de otras comunidades autónomas como Andalucía o Cataluña. Sólo a partir de mayo, las visitas de Madrid vuelven a subir levemente hasta el 36,8\%. Es lógico pensar que el visitante de Madrid consultaba la página web antes de su visita para informarse de la localización, las tarifas y los horarios del museo, pero ante el cierre, este tipo de consultas decaen y otras 
centradas en contenidos educativos digitales, potenciados considerablemente durante ese periodo, hacen aumentar las visitas de otras zonas del Estado.

Por su parte, las consultas de las páginas de actividades y las referentes a la información para la visita (horarios y localización) sufrieron una enorme bajada por la ausencia de actividades presenciales y el cierre del Museo, pero empezó a remontar coincidiendo con la fecha próxima a la reapertura, a principios de junio. Las visitas registradas en la sección 'Comunicación' subieron en los meses del confinamiento respecto a meses anteriores y posteriores. Como dato curioso cabe destacar que, durante el mes de abril, de las 123.136 visitas a páginas, 4.745 fueron al perfil laboral de Fernando Valladares, investigador del MNCN que durante los meses del confinamiento fue muy mediático al ser entrevistado en numerosos programas de radio, televisión y periódicos online.

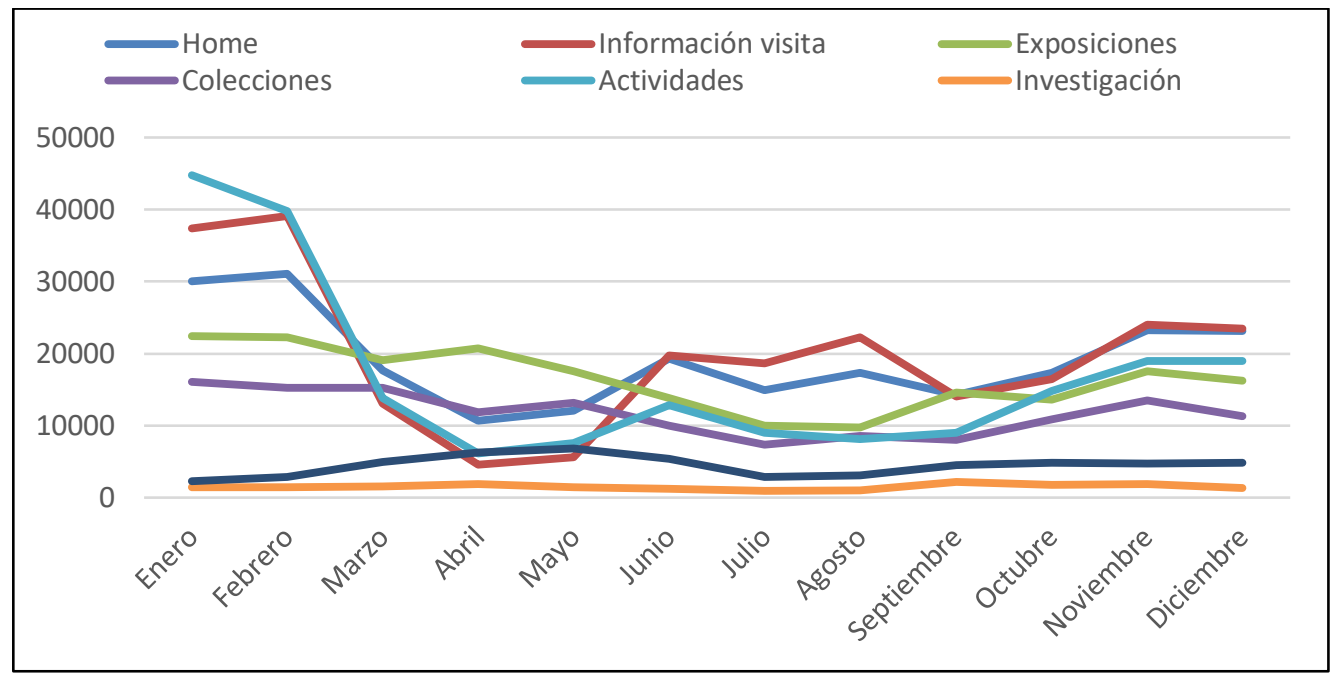

Fig. 3 Evolución de las visitas a páginas web del MNCN por temática

Con respecto a las redes sociales, destaca el crecimiento experimentado en Twitter de marzo a abril* (acumulando 389 nuevos seguidores) y el de Instagram de abril a mayo (508 nuevos seguidores del perfil). Aquí es importante destacar que el crecimiento de la comunidad de seguidores del MNCN es orgánico y que en ningún caso se han promocionado las publicaciones).

\footnotetext{
* Debe recordarse que el 15 de marzo de 2020 se inició el confinamiento domiciliario y el 21 de junio de 2020 finalizó el estado de alarma y se instauró la 'Nueva normalidad'.
} 


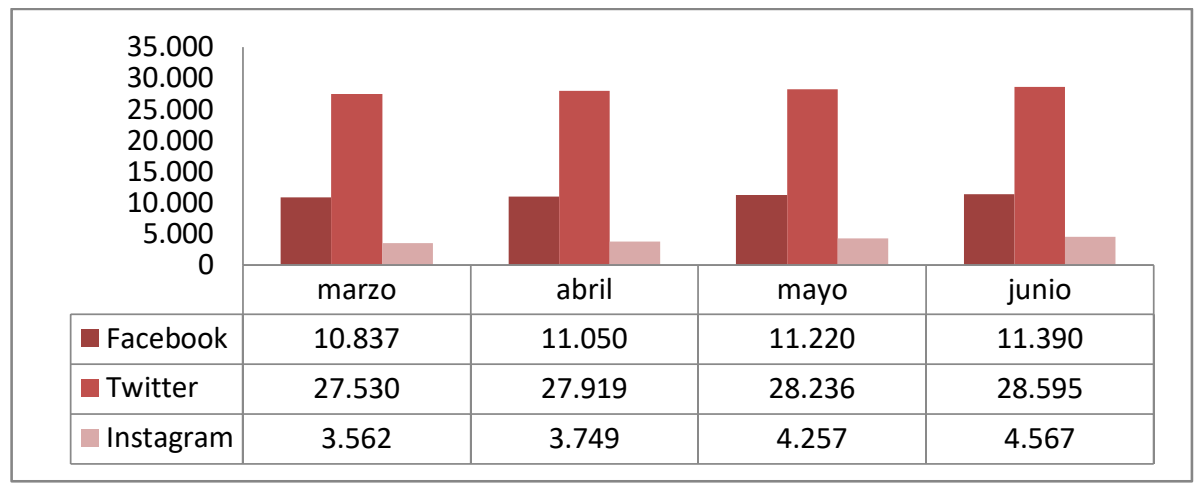

Fig. 3 Seguidores en redes sociales del MNCN de marzo a junio de 2020

Por otro lado, la adquisición 'social' de la página web, es decir la procedente de usuarios que visitan la página a través de redes sociales, cuya media era del $5 \%$ entre octubre 2019 y febrero de 2020, baja inicialmente en marzo hasta el 3,3\% para subir en abril hasta el 7,8\%, coincidiendo con el aumento de nuevos seguidores y mayor interacción de la audiencia digital con el contenido compartido. Muchas de las personas que interactuaron con ese contenido es posible que acabaran accediendo a la página web a través del enlace que siempre se incluyen en las publicaciones. En el mes de mayo este porcentaje se estabilizó en un 5,3\%.

Si tenemos en cuenta los resultados obtenidos a través de la encuesta online, la página web del MNCN (www.mncn.csic.es) y los perfiles de las redes sociales del Museo, fueron los soportes más utilizados que los participantes indicaron haber utilizado más para conectarse con los contenidos del Museo, sobre el resto de las opciones propuestas: otras redes sociales, publicaciones digitales, radio/televisión y el Blog del MNCN.

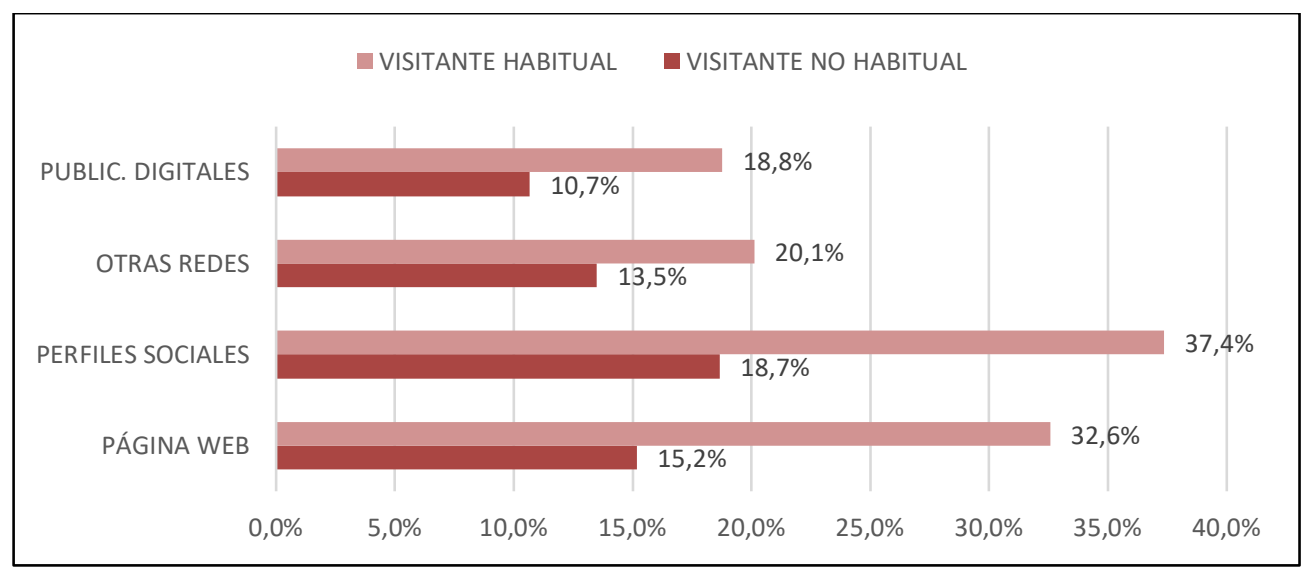

Fig. 4 Búsqueda y seguimiento de la información sobre el MNCN durante el confinamiento (con cierta frecuencia o muy frecuentemente) de los visitantes habituales y no habituales 
Si se considera la búsqueda proactiva de información digital sobre el MNCN o el seguimiento de la misma de manera intencionada durante el periodo de confinamiento, un $48 \%$ de los encuestados afirmaron haber seguido en mayor o menor medida la información publicada por el MNCN. De ellos, un 16\% lo hicieron de forma intermitente y un 7\% regularmente. Los visitantes más habituales, los que habían visitado el Museo en menos de dos años, recordaron haber visto y buscado más información sobre el mismo que los menos habituales, aquellos que nunca lo habían visitado o que lo habían hecho hacía más de dos años.

Durante el confinamiento y cierre de los museos ha sido necesario potenciar el uso y crear nuevos formatos digitales que permitieran llegar a los hogares de los visitantes potenciales. Los visitantes opinaron de forma mayoritaria que estos formatos son interesantes por un tiempo, cuando no se podía acudir presencialmente al Museo (el 83,9\%), que han ayudado a soportar el confinamiento (el 67\%), que pueden ser interesantes para los niños (el 76,4\%) o, incluso, que estos contenidos deberían mantenerse, aunque hubiera visita presencial (el $80,6 \%$ ). Sin embargo, es importante destacar que la mayoría (el 82,4\%) considera que estos recursos nunca podrán sustituir a la visita presencial.

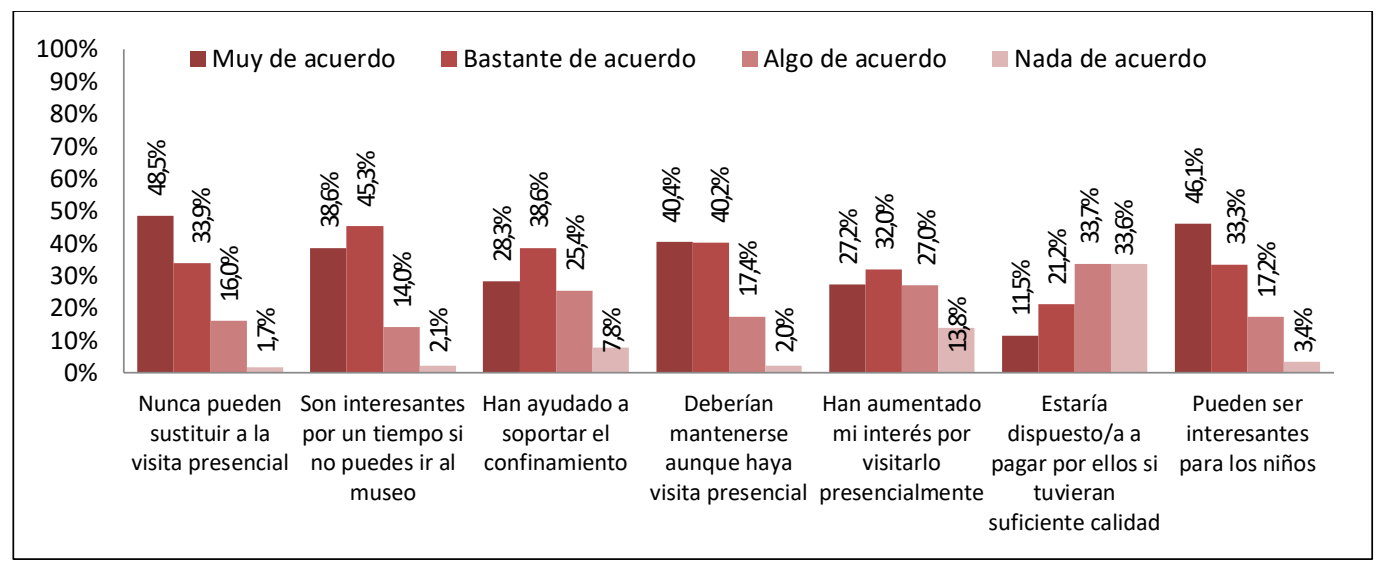

Fig. 5 Grado de acuerdo de los participantes en el cuestionario on line con afirmaciones sobre los recursos digitales

Es interesante destacar que mientras un $32,7 \%$ de los que respondieron el cuestionario se mostraron muy o bastante dispuestos a pagar las actividades digitales y recursos online si tuvieran la suficiente calidad, un 33,6\% se mostró nada de acuerdo con esta iniciativa. 


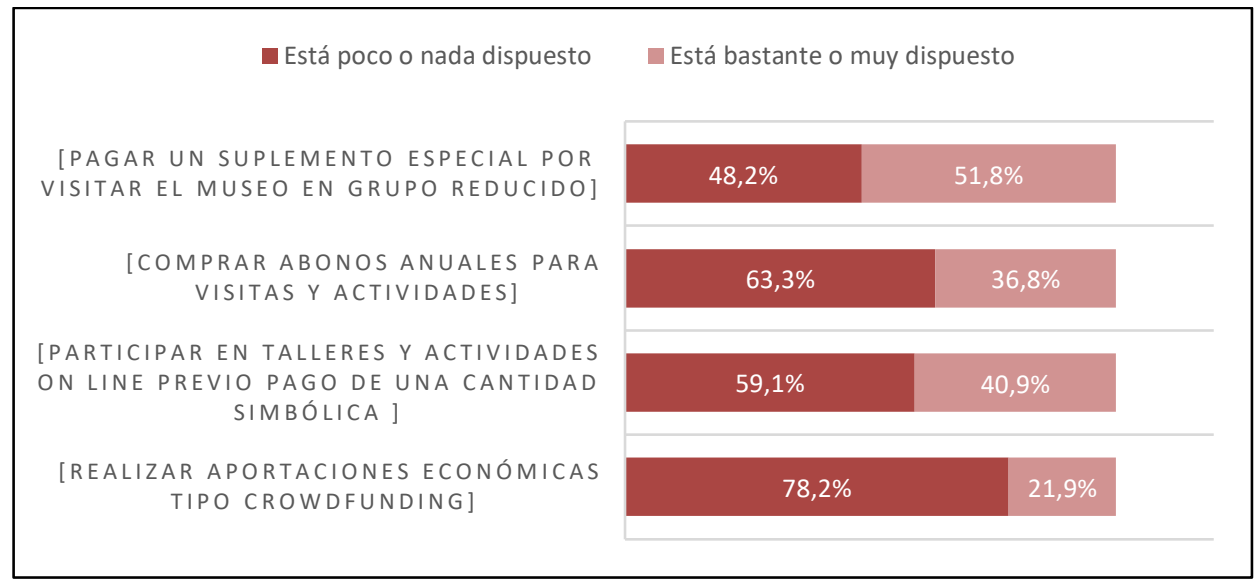

Fig. 6 Disposición a pagar por los recursos digitales de los participantes en el cuestionario online

En el mismo sentido, se evaluó hasta qué punto los usuarios del Museo estarían dispuestos a pagar por algunas actividades antes gratuitas o inexistentes. Se comprobó que el 51,8\% estaría dispuesto a pagar un suplemento especial por visitar el museo en grupos reducidos, el $40,9 \%$ a participar en talleres y actividades online previo pago de una cantidad simbólica y el $36,8 \%$ a comprar abonos anuales para visitas y actividades. Solo un $21,9 \%$ estaría dispuesto a realizar aportaciones económicas tipo crowdfunding.

Valoración de los efectos de las actividades online y presenciales a partir de la reapertura del Museo

El Museo Nacional de Ciencias Naturales permaneció cerrado por la pandemia desde mediados de marzo hasta principios de junio de 2020. La consecuencia de esta situación se plasmó en un descenso considerable en el número de visitas presenciales que pasó de 282.960 en 2019 a 126.289 en 2020.

Sin embargo, como se ha comprobado en otras instituciones museísticas, el seguimiento de las actividades online y la utilización de recursos digitales durante el cierre y, posteriormente en la reapertura se vio incrementado. La web oficial recibió más de 2 millones de visitas a páginas y la comunidad de seguidores en redes sociales de los perfiles del MNCN llego casi a los 50.000 .

Desde la reapertura del Museo en junio de 2020 el seguimiento en redes sociales de los contenidos ofertados ha sido lento pero mantenido tanto en Twitter como en Facebook e Instagram. En diciembre se superó la barrera de los 30.000 seguidores en Twitter. Una cifra a la que ha costado llegar pero que desde enero avanza con buen ritmo, mucho más acelerado que durante 2020. A 25 de febrero de 2021, el número de seguidores de este perfil es de casi 32.000 . 


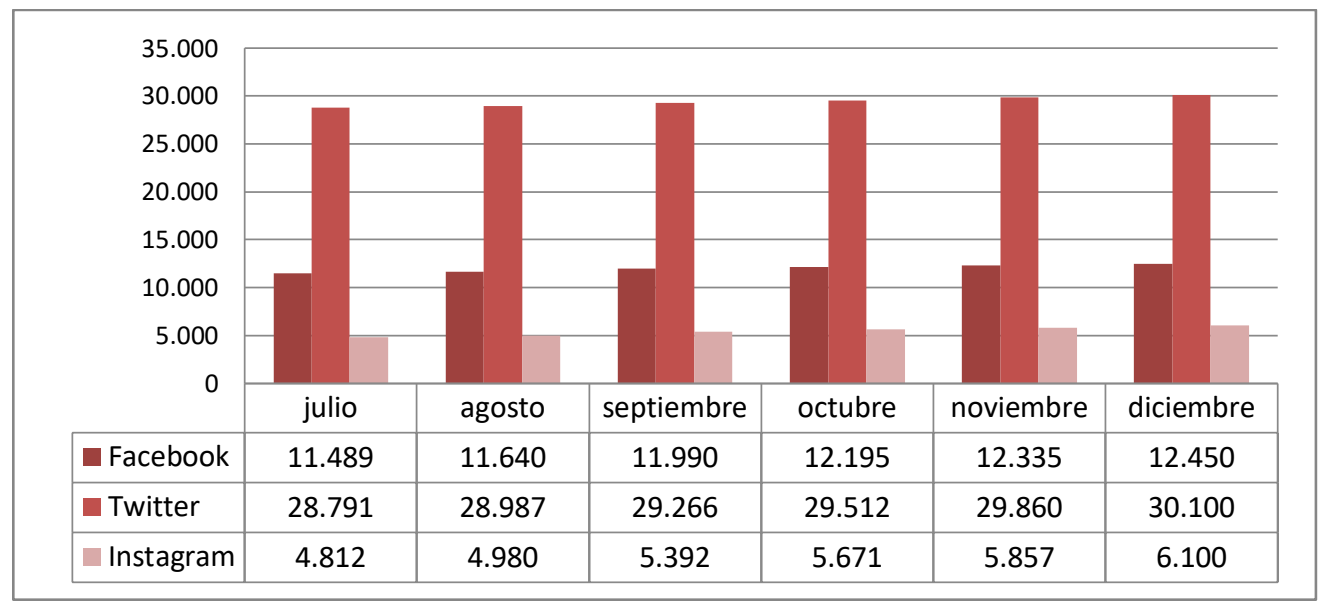

Fig. 7 Seguidores en redes sociales del MNCN de julio a diciembre de 2020

La creación de contenidos online no sólo no han disminuido, a pesar de la apertura a visitantes presenciales, sino que ha ido aumentando durante los últimos meses. Así, se crearon contenidos online, como la gymkhana científica digital que cuenta con casi 3000 visualizaciones, y se realizaron charlas en streaming, como "Desarrollo de una vacuna española contra la COVID-19" impartida por el Doctor Juan García-Arriaza, investigador del Centro Nacional de Biotecnología (CNB-CSIC), con más de 3400 visualizaciones. El MNCN ha seguido, también, realizando webinars [https://www.youtube.com/watch?v=UF5fO6XTrY\&t=76s] $\quad \mathrm{y} \quad$ presentaciones online [https://www.facebook.com/SAMNCN/], [https://www.youtube.com/watch?v=RAuROUoV MRk\&t=181s].

Más allá de las cifras que permiten comprobar cómo los recursos digitales contribuyen a ampliar la audiencia del Museo y hacer posible la interacción con los ponentes, investigadores, conservadores, divulgadores, etc. en tiempo real, y que están disponibles para que el público los consulte en cualquier momento y lugar, no debe olvidarse la capacidad de los mismos para llegar a personas que no pueden participar en las actividades de forma presencial por distintos motivos como problemas de movilidad, discapacidades de todo tipo o encontrarse fuera de Madrid. 


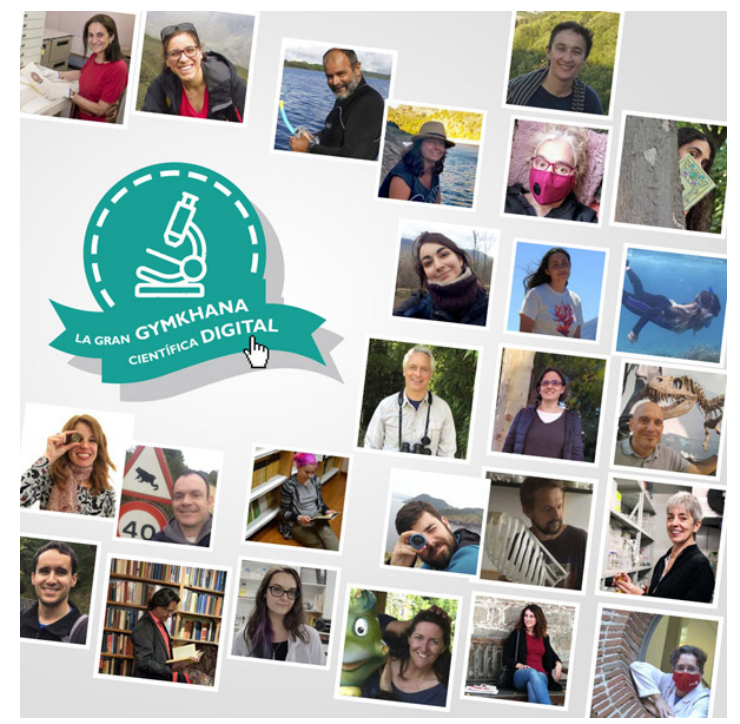

Fig. 8 Gymkhana científica digital, en la que participaron más de 20 investigadores, conservadores y divulgadores del Museo, cuenta con casi 3000 visualizaciones

Debido al éxito de las iniciativas digitales durante el confinamiento, el Museo está trabajando cada vez más por la inclusión de este formato, en combinación con el presencial, en todos sus eventos. El caso más reciente es el del Día Internacional de la Mujer y la Niña en la Ciencia, celebrado el 11 de febrero de 2021, que lleva más de 674 visualizaciones, unas cifras impensables de alcanzar para el Museo de forma presencial. En la actualidad se trabaja, también, en el diseño de actividades digitales para los centros educativos que no pueden desplazarse al Museo, intentando ofrecer una experiencia virtual en la que los alumnos puedan interactuar de forma directa con los educadores y educadoras y realizar un taller como si se encontraran en las propias aulas educativas del Museo.

Tabla 2 Contenidos digitales desarrollados en el MNCN desde marzo de 2020

\begin{tabular}{|l|l|l|}
\hline Recursos & \multicolumn{1}{l}{ Visualizaciones } & \multicolumn{1}{l|}{ Enlace } \\
\hline $\begin{array}{l}\text { Gymkhana científica digital } \\
\text { Charla 'Desarrollo de una vacuna } \\
\text { española contra la COVID-19' }\end{array}$ & 3000 & https://bit.ly/3ssKUVp \\
\hline Webinars y conferencias & 2552 & https://bit.ly/386HkJ1 \\
\hline Presentaciones online & 1462 & $\begin{array}{l}\text { https://bit.ly/2ZRIOgF } \\
\text { https://bit.ly/2ZSOhc8 } \\
\text { https://bit.ly/2ZUh6Vi }\end{array}$ \\
\hline
\end{tabular}




\begin{tabular}{|l|l|l|}
\hline & & https://bit.ly/2ZRLyQ3 \\
\hline Recursos digitales & 3014 & https://bit.ly/2ZSeSWx \\
\hline $\begin{array}{l}\text { Recursos 'Día Internacional de los } \\
\text { Museos' }\end{array}$ & 1.558 & https://bit.ly/3bHwLg9 \\
\hline $\begin{array}{l}\text { Mesa redonda 'MNCN en } \\
\text { femenino' }\end{array}$ & 674 & https://bit.ly/2ZPNubR \\
\hline $\begin{array}{l}\text { Guías multimedia accesibles } \\
\text { Publicaciones audiovisuales sobre } \\
\text { proyectos de accesibilidad }\end{array}$ & 1516 & https://bit.ly/3r3tQFi \\
\hline \begin{tabular}{l} 
Recursos didácticos accesibles \\
https://bit.ly/37MMDwl \\
https://bit.ly/3pUb81u \\
\hline
\end{tabular} & 174 & https://bit.ly/3ktktwa \\
\hline
\end{tabular}

Los resultados obtenidos, durante el cierre, sobre la disposición de los visitantes a volver a visitar e implicarse en las actividades del Museo y el estudio de las condiciones que ofrecían más confianza ante la pandemia, permitió poner en marcha, con las medidas de seguridad adecuadas, algunas actividades tradicionales del Museo. Así, durante el verano de 2020 y, bajo unas estrictas medidas de seguridad y control, los resultados del estudio aportaron los datos necesarios para plantear actividades presenciales tradicionales en el Museo como 'Museo de Verano, el campamento urbano del MNCN', al que asistieron 487 participantes. El MNCN también participó en eventos como la Noche Europea de las investigadoras y los investigadores (con 150 asistentes presenciales y 1500 visualizaciones en el evento online) o la Semana de la Ciencia (con 242 asistentes). 


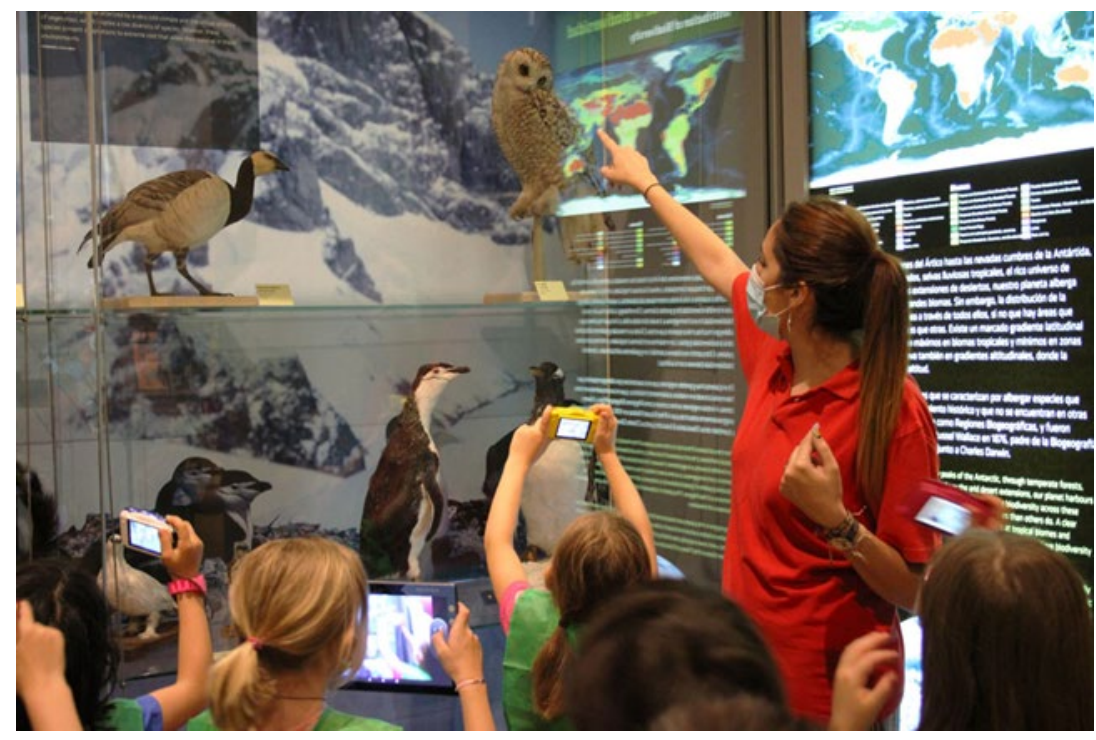

Fig. 9 Participando en una actividad infantil durante el verano de 2020

Los visitantes presenciales del Museo, que volvieron a partir de su reapertura, mostraron una gran satisfacción con las medidas de seguridad adoptadas y corroboraron la utilización de las consultas digitales para seguir conectados con la Institución e informarse. Así se comprobó en las entrevistas que se llevaron a cabo el día de la reapertura entre el público asistente.

\section{Conclusiones}

Uno de los resultados más interesantes de este estudio es la acogida que tuvieron los contenidos digitales durante el periodo de confinamiento. Concretamente, es interesante el seguimiento que realizaron los visitantes de estos recursos de forma proactiva, es decir, con la intención de estar informados sobre los diferentes contenidos que el MNCN ha ido produciendo para sus redes sociales y su página web. Como se ha hallado en otros estudios, los visitantes más habituales, aquellos que habían realizado varias visitas presenciales en los últimos 2 años, fueron los que más información recordaron haber visto y los que habían hecho un seguimiento más frecuente de sus contenidos digitales.

Podemos concluir que durante el confinamiento y cierre de los museos ha sido necesario potenciar el uso y crear nuevos formatos digitales que han permitido llegar a los hogares de los ciudadanos, algunos de ellos visitantes potenciales. Pero también, puede derivarse de los resultados obtenidos en este trabajo, que el seguimiento de los recursos digitales ha mantenido, potenciado o despertado el deseo de la visita presencial, por la vinculación cognitiva y emocional que estos recursos son capaces de generar en las audiencias. 
Como muy bien los visitantes expresaron en el cuestionario utilizado para este trabajo, los formatos digitales son interesantes por un tiempo, cuando no se pudo acudir presencialmente al Museo, ayudando a soportar el confinamiento, pero, por otro lado, aunque difícilmente puedan sustituir a la visita presencial, la mayoría de los visitantes están de acuerdo en que deberían mantenerse.

Como se ha comprobado en otras instituciones museísticas, la web y la comunidad de seguidores en las redes sociales, el seguimiento de las actividades online y la utilización de recursos digitales durante el cierre $\mathrm{y}$, posteriormente en la reapertura se vio considerablemente incrementado. Por este motivo los proyectos en los que se contempla la producción de vídeos, recursos digitales y actividades online serán una tendencia en aumento en los museos, en combinación con la programación de actividades presenciales.

Escuchar a los visitantes habituales y potenciales, presenciales y digitales, a través de metodologías cruzadas que relacionen datos cuantitativos y cualitativos obtenidos a través de procedimientos presenciales, métricas digitales o sistemas de evaluación online o a través de redes sociales, permite recibir sugerencias relativas a la organización y programación del museo. En estas consultas se valoran las actividades ya existentes, se mide la tendencia que marcan los usuarios para determinar hacía donde se deben encaminar las nuevas propuestas y es una estupenda forma de comenzar a empoderar progresivamente al visitante de museos.

\section{Referencias}

DUDO, A. y BESLEY, J. C. (2016). "Scientists' prioritization of communication objectives for public engagement”. PloS one, v. 11, n. 2, e0148867. https://doi.org/10.1371/journal.pone.0148867

LÓPEZ GARCÍA-GALLO, P. (2015) "Los programas públicos del MNCN. Propuestas didácticas de divulgación científica para dinamizar el Museo". Revista de museología, No.64. Asociación de Museólogos de España,

LÓPEZ GARCÍA-GALLO, P. (2017) "La divulgación científica en el MNCN. El reto de innovar en un museo con más de dos siglos de historia”. Revista de Didácticas Específicas, $\mathrm{n}^{\circ} 17$, pp. 109-122 Didácticas Específicas, ISSN: 1989-5240 122 www.didacticasespecificas.com

PÉREZ-SANTOS, E. (2009) “Metodología básica de la investigación de público en museos: áreas de actuación, variables implicadas, tipos de investigaciones y técnicas utilizadas". $\begin{array}{llllll}\text { Revista } & \text { Mus-A. } & \text { Vol. } & 10 & \text { pp. } & 48-57\end{array}$ http://www.juntadeandalucia.es/cultura/museos/media/docs/PORTAL_musa_10_ok.pdf

PÉREZ-SANTOS, E. (2018) Sistema de recolección de datos de público de museos: Cuestionario básico y manual de aplicación. Ibermuseos.

http://www.ibermuseos.org/acciones/observatorio-iberoamericano-de-museos/sistema-derecoleccion-de-datos-de-publico-de-museos/ 
PÉREZ-SANTOS, E. (2019) Investigaciones de públicos en los museos: Evolución histórica, problemas actuales y retos futuros. En Fernando Bayón y Jaime Cuenca (eds.): Públicos en transformación. Una visión interdisciplinar de las funciones, experiencias y espacios del público actual de los museos, pp 19-27. Dykinson.

PÉREZ-SANTOS, E. (2020) Best Practices in Visitors Studies. The Permanent Laboratory of Museum Audiences (Spain) En:. Alejandra Alonso Tak and Ángel Pazos-López Socializing Art Museums. Rethinking the publics experiencies. De Gruyter. https://doi.org/10.1515/9783110662085-003

VALERO, J.M. y GARCÍA, A, (2018). "La importancia de comunicar la ciencia en el nuevo ecosistema móvil”. Divulgación científica e innovación mediática. Comunicar la ciencia en el ecosistema móvil. Egregius Ediciones. ISBN: 9788417270704

VILLAESPESA, E. (2018) "Evaluación de la estrategia digital en los museos: métodos y herramientas para web, redes sociales y móviles" Periférica: Revista para el análisis de la cultura y el territorio, ISSN 1577-1172, Nº 19, 2018, págs. 38-53 